\title{
Possible protective effect of ethanol on acute methanol intoxicated retina in rats: A histological and ultrastructural study
}

\section{Original Article}

\author{
Rabab Ahmed Rasheed ${ }^{1}$, Magdy Fouad Youakim ${ }^{2}$ and Mohamed Ahmed Yehia \\ ${ }^{1}$ Department of Histology, Faculty of Medicine, October 6 University, Egypt
}

${ }^{2}$ Department of Anatomy and Embryology, Faculty of Medicine, Cairo University, Egypt

${ }^{3}$ Department of Forensic Medicine and Clinical Toxicology, Faculty of Medicine, October 6 University, Egypt

\begin{abstract}
Background: Methanol poisoning frequently occurs via oral ingestion of adulterated spirits as ethanol substitutes. Retina is particularly susceptible to the toxic effects of methanol. Ethanol is frequently used for treatment where prompt management is crucial to avoid irreversible ocular damage.

Aim of the work: The aim of this work is to study the histological and ultrastructural effect of methanol intoxication on the retina, as well as the ability of ethanol to protect against methanol induced retinal damage.

Materials and Methods: Thirty adult male albino rats, were used in the study. Rats were divided into three groups ( $\mathrm{n}=10$ per group): Group 1 (Control group); Group 2 (Methanol treated group) and Group 3 (Methanol + Ethanol treated group). Rats were sacrificed after 24 hours of methanol administration. Retinae were examined histologically and ultrastructurally.

Results: Histological and ultrastructure examination of the retina of rats treated with methanol showed marked edema of all layers, disorganized nuclear layers and decreased number of ganglion cells. Electron microscopic examination revealed fragmented outer segment, disorganized inner segment. Administration of ethanol in methanol intoxicated rats resulted in preservation of retinal structure with marked attenuation of methanol induced retinal damage.

Conclusions: Methanol poisoning induces damage of the retina. Administration of ethanol protects the retina and attenuates histopathological changes induced by methanol.
\end{abstract}

Key Words: Ethanol, Methanol intoxication, Retina, Ultrastructure

Revised: 7 October 2017, Accepted: 8 January 2018

Corresponding Author: Rabab Ahmed Rasheed, Department of Histology, Faculty of Medicine, October 6 University, Egypt, Tel.: +201001022257, E-mail: rasheedrabab@gmail.com

ISSN: 2536-9172, Vol.1, No.2

\section{INTRODUCTION}

Methanol is also known as methyl alcohol. It has the chemical formula $\mathrm{CH}_{3} \mathrm{OH}$. It is extremely toxic for human. Methanol poisoning most frequently occur via oral ingestion of adulterated spirits as ethanol substitutes ${ }^{[1]}$. Because of its resemblances in both appearance and odor to ethanol, it is difficult to distinguish between the two. Methanol poisoning may occur as large outbreaks which typically occur in developing countries and have been related with high morbidity and mortality ${ }^{[2]}$.

The absorption of methanol after oral administration is rapid and peak methanol concentration occurs in $30-60$ minute $^{[3]}$. In the liver, methanol is metabolized to formaldehyde by alcohol dehydrogenase enzyme. Formaldehyde is converted to formic acid by aldehyde dehydrogenase enzyme. Formic acid formed is the toxic metabolite responsible for the noxious effects of methanol intoxication $^{[4,5]}$. Formic acid inhibits mitochondrial cytochrome oxidase enzyme of the electron transport chain causing symptoms of hypoxia at cellular level along with metabolic acidosis ${ }^{[6,7]}$. Moreover, methanol causes lipid peroxidation and depletes free radical scavenger system ${ }^{[8]}$. There is a latent period lasting from 12 to 24 hours depending on the dose of methanol ingested, following which metabolic acidosis develops and vision becomes impaired $^{[3]}$.

Ocular tissues are particularly vulnerable to the toxic effects of methanol ${ }^{[9]}$. Visual impairment ranges from blurred vision, visual fields defect to blindness, where eye examination after methanol poisoning may show retinal and optic disc edema as well as hyperemia ${ }^{[10,11]}$.

Visual injury and other complications of methanol toxicity could be prevented with prompt medical management. Methanol toxicity can be treated by ethanol, which decreases the action of alcohol dehydrogenase on methanol by means of competitive inhibition, so methanol will be excreted by the kidneys rather than being converted into toxic metabolite. Further treatment includes correction of metabolic acidosis by sodium bicarbonate, administration of folinic acid to stimulate formic acid metabolism, in addition to hemodialysis to remove methanol and formic acid from the blood ${ }^{[3]}$. 
Although most of the reviewed literatures extensively studied methanol toxicity on the eye clinically, only few studies discussed histological and ultrastructural changes on the retina.

\section{AIM OF STUDY:}

The aim of this work was to study the histological and ultrastructural effect of methanol intoxication on the retina. Moreover, the current study aimed to assess the efficacy of ethanol in treating histopathological changes induced by methanol in the retina.

\section{PATIENTS AND METHODS}

\section{Chemicals:}

Methanol (45\%) and Ethanol (70\%) were obtained from El-Gomhoreya Chemicals Company, Egypt.

\section{Animals and experimental protocols:}

Thirty adult male albino rats (Sprague-Dawley) weighing 180-200g, were used in the present work. Rats were bred and maintained in an air-conditioned room under specific pathogen free condition and were subjected to a 12:12-h day light/darkness cycle with free access to food and water. All the ethical protocols and guidelines for animal handling and treatment were followed. Rats were divided into three groups ( $\mathrm{n}=10$ per group) as follows:

-Group 1 (Control group): rats received standard diet only.

-Group 2 (Methanol treated group): rats were given methanol $45 \%$ orally via gastric intubation at a dose of $3 \mathrm{~g} / \mathrm{kg}$ body weight ${ }^{[12]}$.

-Group 3 (Methanol + Ethanol treated group): animals were given methanol as in Group 2, then ethanol 70\% was given via gastric intubation at a dose of $0.5 \mathrm{~g} / \mathrm{kg}^{[13]}$ four hours later as formaldehyde levels in CSF present gradually increasing trends which began at 3 hours after methanol administration ${ }^{[14]}$.

\section{Histological and ultrastructural assessment of rats'}

\section{retinae:}

All rats were anesthetized with ether and sacrificed by cervical dislocation after 24 hours of methanol administration. The right eyes of the rats were enucleated carefully. The anterior segment and vitreous were removed, then full-thickness pieces of eye wall were dissected from posterior pole and were sliced into two parts horizontally passing through the optic nerve.

The first part was fixed in 10\% formalin solution and embedded in paraffin wax. Sections of $5 \mu \mathrm{m}$ thickness were cut on a microtome and stained with haematoxylin and eosin $(\mathrm{H} \& \mathrm{E})$ for general histologic examination ${ }^{[15]}$.

The second part was divided into $1 \mathrm{~mm}$ slices and was processed for transmission electron microscopy. Small blocks of tissue were fixed in $2.5 \%$ glutaraldehyde in $0.1 \mathrm{M}$ sodium cacodylate buffer at $4{ }^{\circ} \mathrm{C}$ for $6 \mathrm{~h}$, dehydrated in ascending grades of ethyl alcohol, embedded in EM bed 812 resin (EM bed 812 kit, Electron Microscopy Sciences, Hatfield, PA, USA) and polymerized at $60^{\circ} \mathrm{C}$ for 24 hours. To allow selection of the appropriate tissue area before proceeding to TEM, semi-thin sections were prepared at approximately $0.5-1 \mu \mathrm{m}$ thickness using glass knives and Leica Ultracut UCT ultra microtome and stained with $1 \%$ toluidine blue in $1 \%$ borax. Ultrathin sections (75-90 nm thick) collected on 300 mesh copper grids were contrast stained with uranyl acetate and lead citrate. The sections were examined in a JEOL transmission electron microscope (JEM-1400, JEOL, Tokyo, Japan) at $80 \mathrm{kV}$. Images were captured by a side-mounted CCD digital camera (AMT Optronics, 1632 x 1632 pixel format). The transmission electron microscopy was done in FARP TEM lab, Faculty of Agriculture, Cairo University-Research Park (CURP) ${ }^{[16]}$

\section{RESULTS}

Histology of the retina using haematoxylin and eosin stain:

Light microscopic examination of the retina of Group 1 showed normally arranged retinal layers formed of outer segment and inner segment of photoreceptors, outer nuclear layer, outer plexiform layer, inner nuclear layer, inner plexiform layer and ganglion cell layer [Fig. 1].

Retina of Group 2 showed generalized swelling of whole layers. The inner segment of photoreceptors showed obvious interstitial edema. The outer nuclear layer was thickened with spaced nuclei of photoreceptors. Outer plexiform layer was thickened. The inner nuclear layer was swollen and irregular with markedly separated nuclei. Inner plexiform layer showed marked edema Ganglion cell layer was also swollen with decreased number of ganglion cells with vacuolated cytoplasm. Some ganglionic cells showed apoptotic changes [Fig. 2].

These histopathological changes were ameliorated in Group 3 when compared to Group 2. The inner segment layer of photoreceptors exhibited mild edema. The nuclei of outer nuclear layer were more packed and organized. Outer plexiform layer was thin. The inner nuclear layer showed less edema and more regular arrangement. The inner plexiform layer showed mild edema. The ganglion cells were relatively more in number and their cytoplasm appeared less vacuolated [Fig. 3] 


\section{Histology of the retina using toluidine blue stain:}

Semi-thin sections examination of retina of Group 1 showed regular arrangement and appearance of retinal layers [Fig. 4].

Retina of Group 2 showed markedly swollen outer nuclear layer. The nuclei of outer nuclear layer were widely separated. The outer plexiform layer was swollen. The cells of inner nuclear layer were swollen; their cytoplasm was vacuolated with marked thickening of inner plexiform layer [Fig. 5].

Retina of Group 3 showed generally less edema of retinal layers. The inner segment of photoreceptors showed mild edema. The outer nuclear layer was more organized with mild edema. The outer plexiform layer was less thickened. The cells of inner nuclear layer were less swollen with vacuolated cytoplasm. [Fig. 6].

\section{Ultrastructure examination of the retina:}

Electron microscopic examination of retina of Group 1 showed normal photoreceptors layer. Outer segment presented normal morphology and inner segment

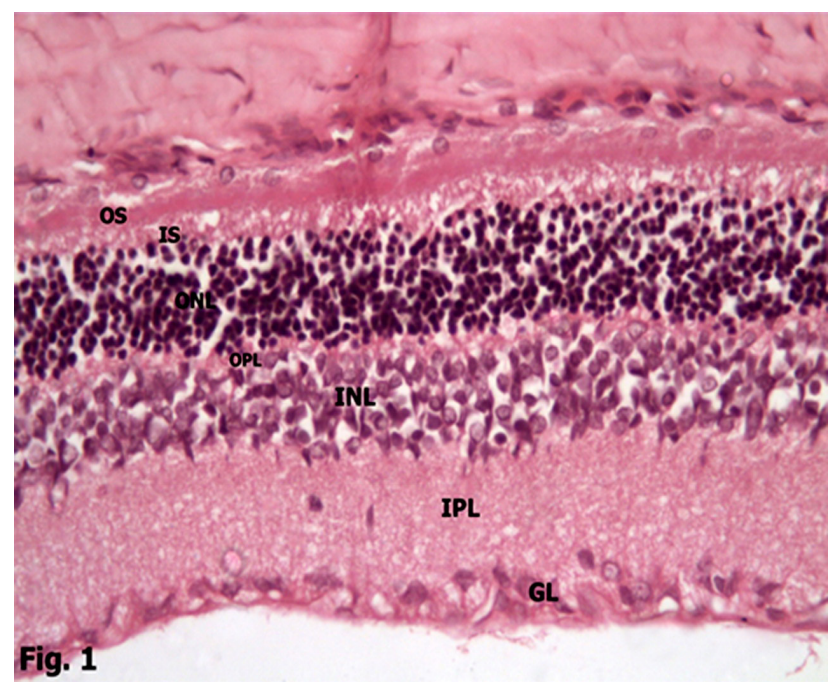

Fig. 1: Photomicrograph of retina of Group 1 showing photoreceptors with outer segment [OS], inner segment [IS], outer nuclear layer $[\mathrm{ONL}]$, outer plexiform layer [OPL], inner nuclear layer [INL], inner plexiform layer [IPL] and ganglion cell layer [GL].

$(\mathrm{H} \& \mathrm{E}$ x 400) contained numerous round and oval mitochondria. The outer limiting membrane was well defined. Nuclei of cones of outer nuclear layer had heterogeneous chromatin appearance while rods had smaller, darker and centrally located chromatin [Fig. 7].

Retina of Group 2 showed that the photoreceptors layer presented the most profound changes in the form of fragmented outer segment and disorganized vacuolated inner segment. Mitochondria in inner segment were few and of abnormal morphology. The outer limiting membrane was interrupted. There was marked spacing between nuclei in outer nuclear layer. Some shrunken nuclei were noticed [Figs. 8, 9, 10, 11].

Retina of Group 3 revealed generally healthier histological features of photoreceptors. The outer segment presented normal morphology and was more organized. The inner segment was more regular with less vacuolization. Mitochondria in inner segment exhibited normal morphology but still fewer than normal. The outer limiting membrane was reformed in some areas. Nuclei of outer nuclear layer appeared more regular with centrally located heterogeneous chromatin. Few shrunken nuclei were also noticed [Figs. 12 \& 13].

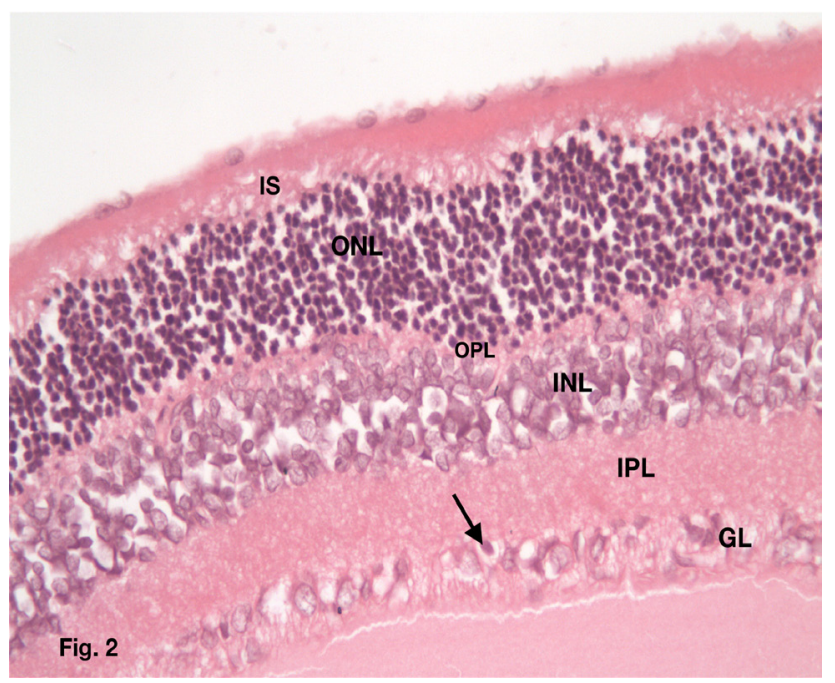

Fig. 2: Photomicrograph of a retina of Group 2 showing generalized swelling of whole layers. The inner segment [IS] shows interstitial edema. The outer nuclear layer [ONL] is thickened and disorganized. Outer plexiform layer [OPL] is thickened. The inner nuclear layer [INL] is swollen and irregular with widely separated nuclei. The inner plexiform layer [IPL] shows marked edema. The ganglion cell layer [GL] is also swollen with relatively decreased number of ganglion cells. Some ganglionic cells show apoptotic changes [arrow]. (H\&E x400) 


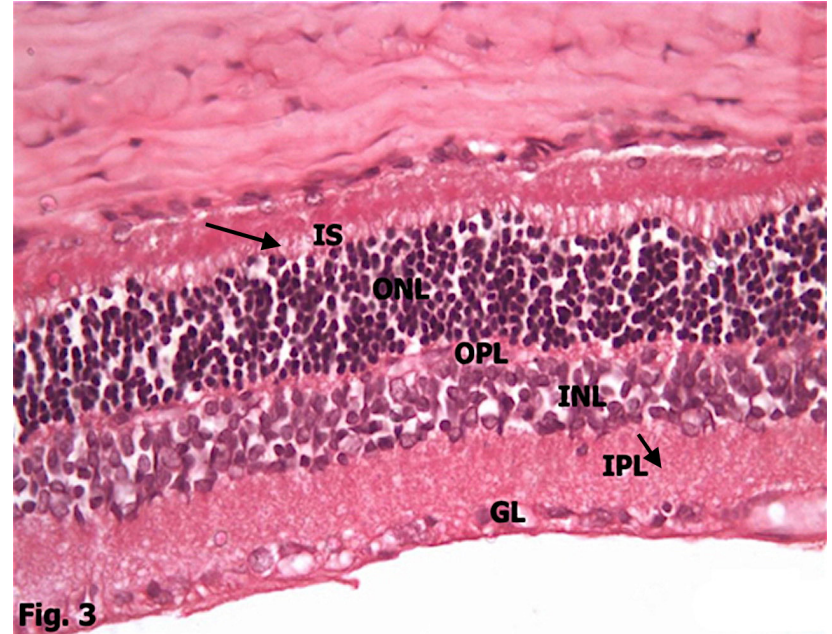

Fig. 3: Photomicrograph of a retina of Group 3 showing the inner segment layer of photoreceptors [IS] exhibiting mild edema (long arrow). The nuclei of outer nuclear layer [ONL] are organized. Outer plexiform layer [OPL] is thin. The inner nuclear layer [INL] shows regular arrangement. The inner plexiform layer [IPL] shows mild edema (short arrow). The ganglion cells [GL] shows unremarkable changes.

$(\mathrm{H} \& \mathrm{E} \times 400)$.

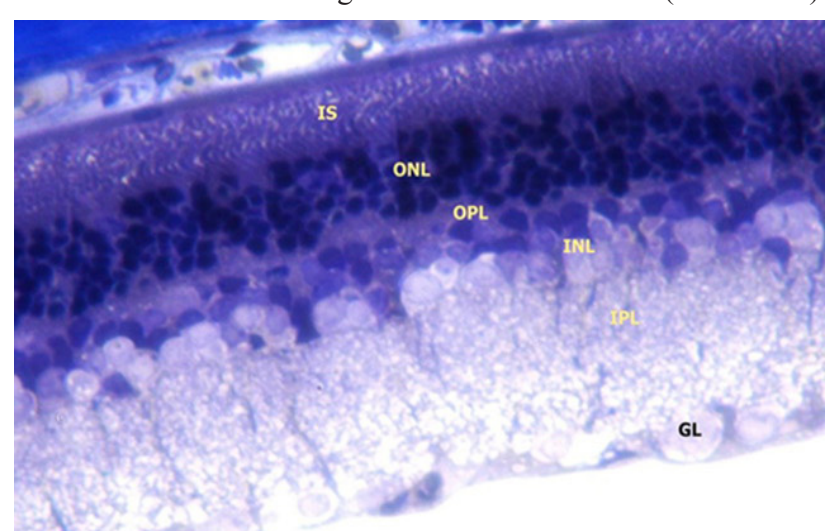

\section{Fig. 4}

Fig. 4: Photomicrograph of a retina of Group 1 showing photoreceptors with inner segment [IS], outer nuclear layer [ONL], outer plexiform layer [OPL], inner nuclear layer [INL], inner plexiform layer [IPL] and ganglion cell layer [GL].

(Toluidine blue $\mathrm{x} 1000$ )

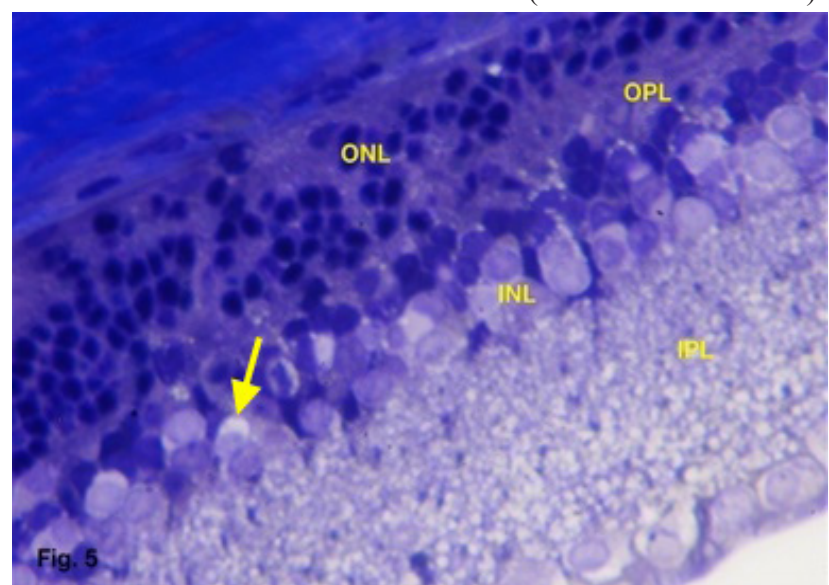

Fig. 5: A photomicrograph of a retina of Group 2 showing markedly dispersed, widely separated nuclei of outer nuclear layer [ONL]. The outer plexiform layer [OPL] is swollen. The cells of inner nuclear layer [INL] show vacuolated cytoplasm [arrow]. The inner plexiform layer [IPL] is markedly thickened. (Toluidine blue x1000).

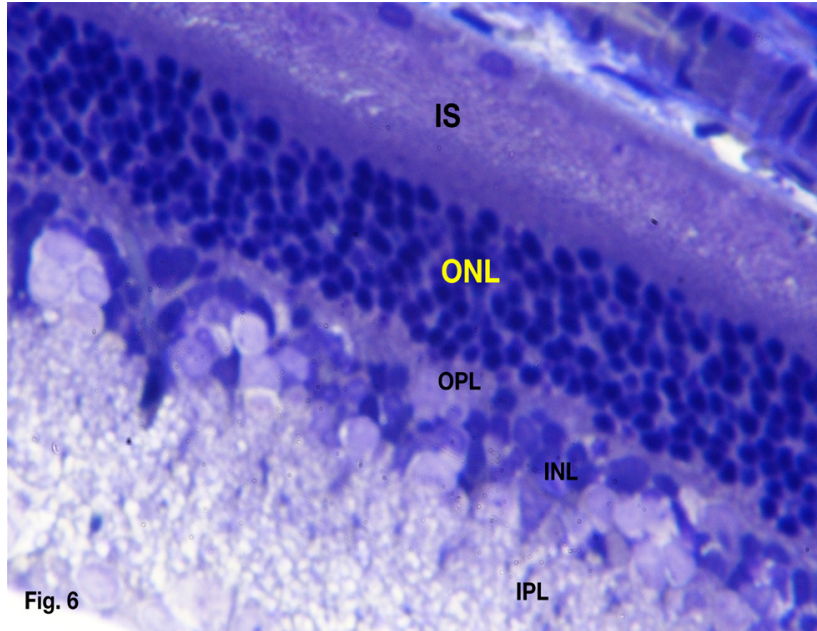

Fig. 6: A photomicrograph of a retina of Group 3 showing mild edema in the inner segment of photoreceptors [IS]. The outer nuclear layer [ONL] is organized. The outer plexiform layer [OPL] shows slight thickening. The cells of inner nuclear layer [INL] are mildly swollen. The inner plexiform layer [IPL] shows mild edema

(Toluidine blue $\mathrm{x} 1000$ )

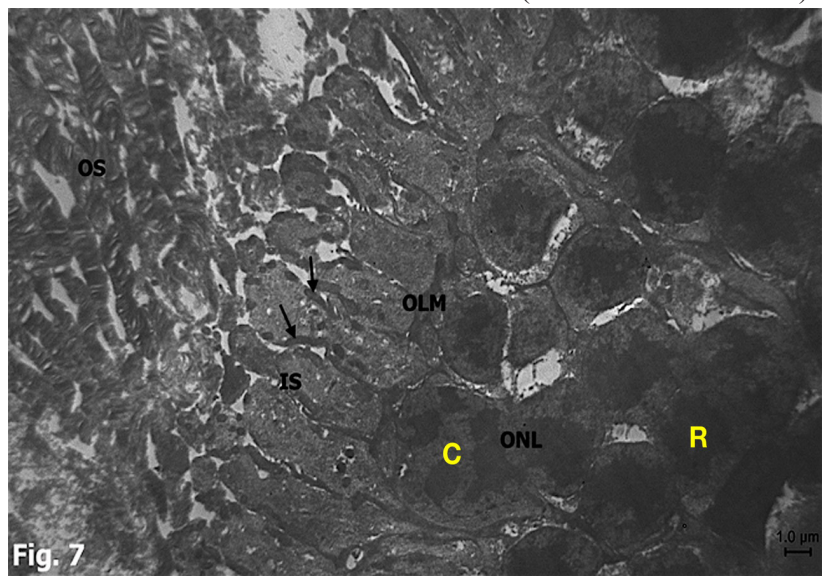

Fig. 7: An electron micrograph of ultrathin section of photoreceptors of Group 1 showing outer segment [OS], inner segment [IS] with numerous round and oval mitochondria [arrow], clear outer limiting membrane [OLM] and outer nuclear layer [ONL]. Cones [C] have different appearance of chromatin compared to rods $[R]$ which have smaller, centrally located dark chromatin

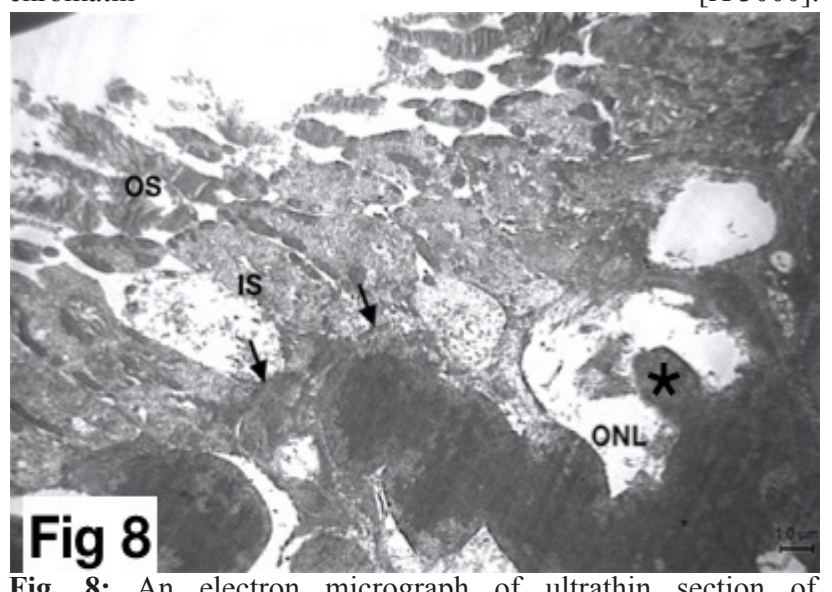

Fig. 8: An electron micrograph of ultrathin section of photoreceptors of Group 2 showing fragmented outer segment [OS], disorganized inner segment with few mitochondria [IS], interruption of outer limiting membrane (arrows) and marked spacing between nuclei of outer nuclear layer [ONL] with a clearly seen shrunken nucleus (star).

[X 4000]. 


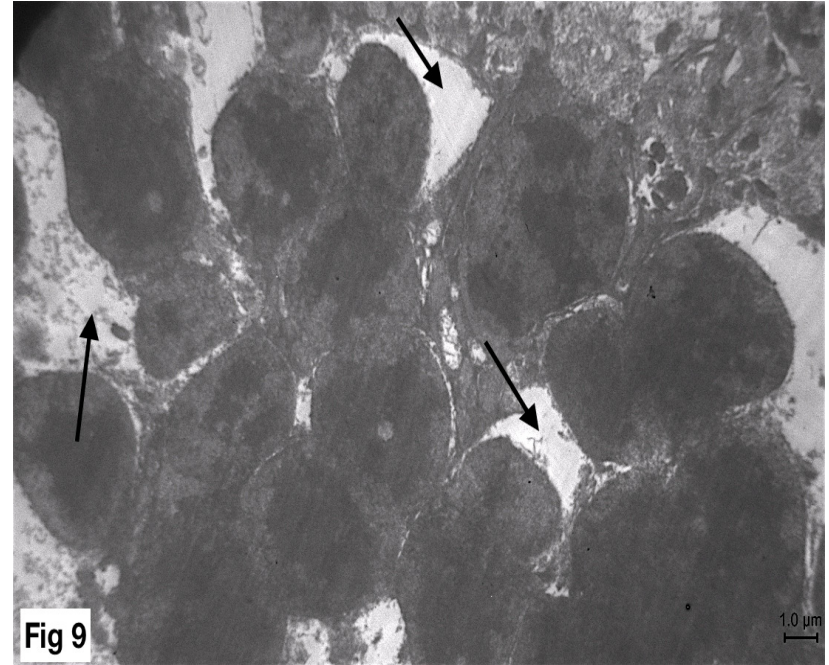

Fig. 9: An electron micrograph of ultrathin section of photoreceptors of Group 2 showing marked spacing between nuclei of outer nuclear layer [arrows].

[X 4000].

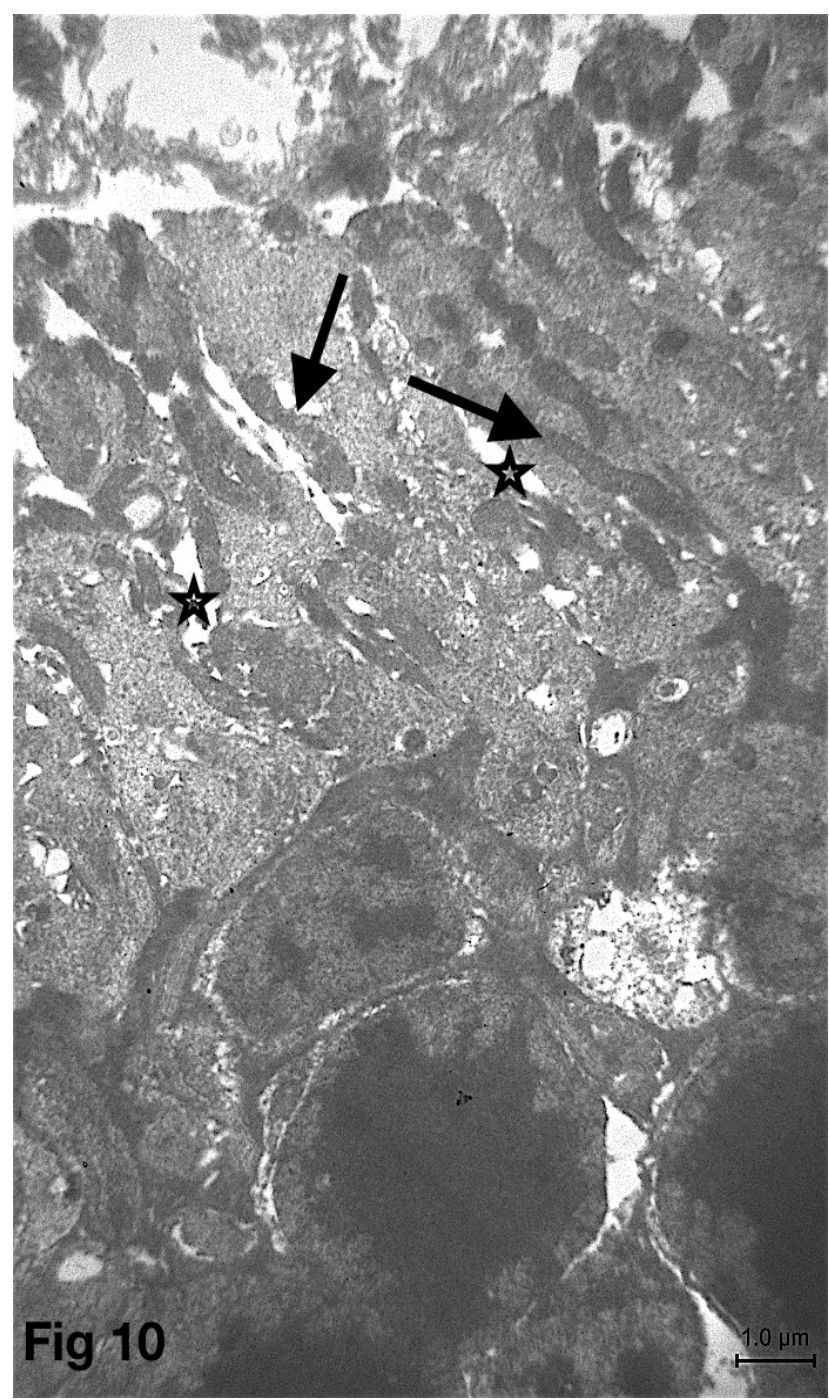

Fig. 10: An electron micrograph of ultrathin section of photoreceptors of Group 2 showing vacuolization of inner segment (stars). The mitochondria were few with abnormal morphology (arrows).

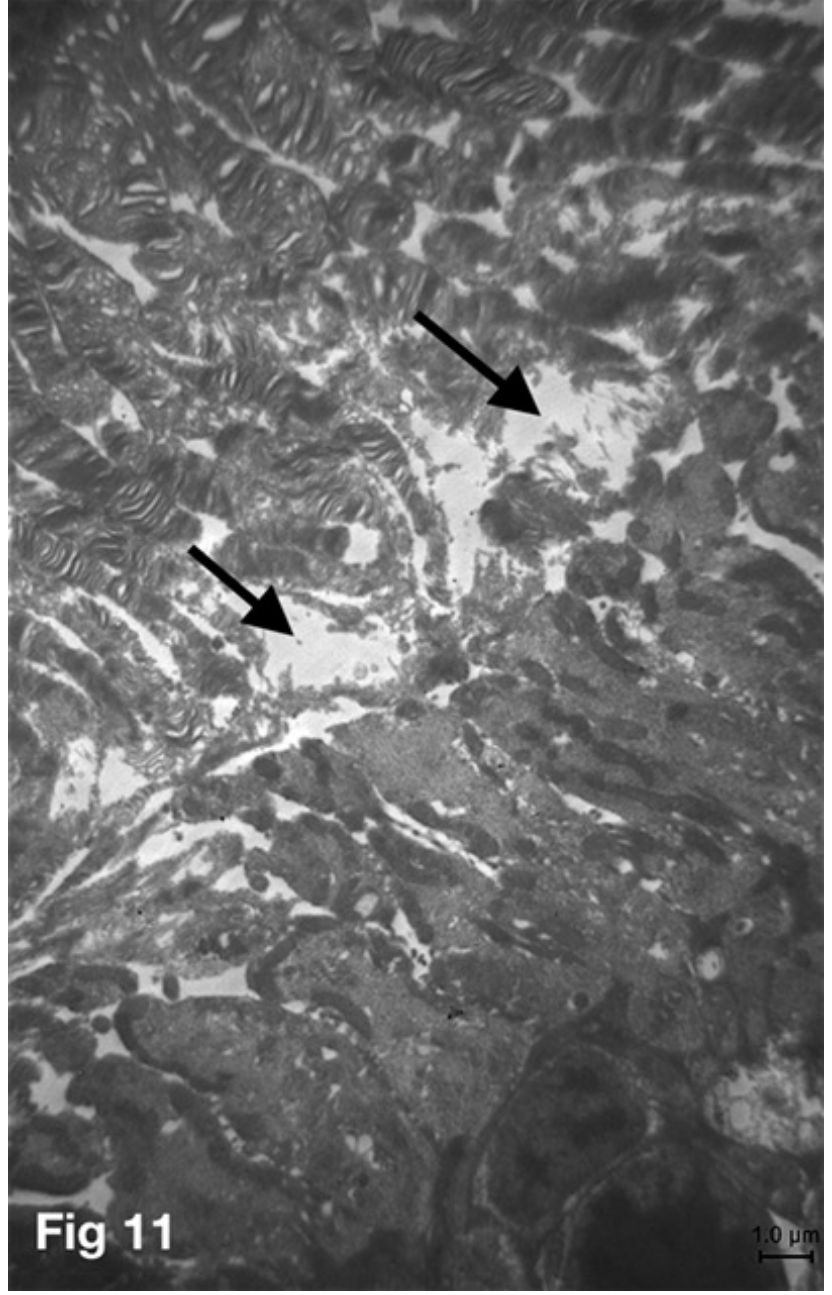

Fig. 11: An electron micrograph of ultrathin section of photoreceptors of Group 2 showing areas of fragmented outer segment [arrows]

[X 4000]. 


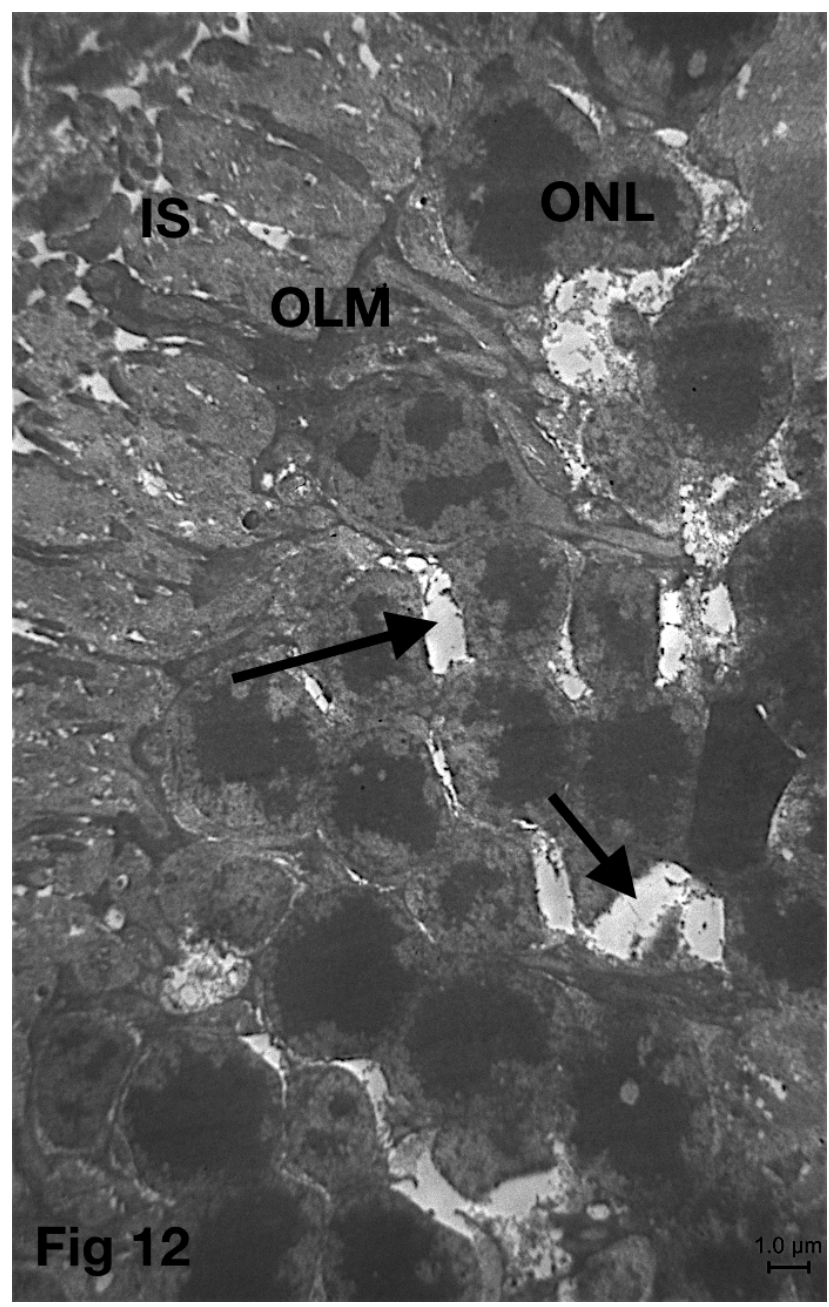

Fig. 12: An electron micrograph of ultrathin section of photoreceptors of Group 3 showing improved histological features, regular inner segment with areas of vacuolization [IS] and outer nuclear layer [ONL] with reformed outer limiting membrane [OLM]. Wide spacing between nuclei of ONL could still be seen (arrows).

[X 3000].

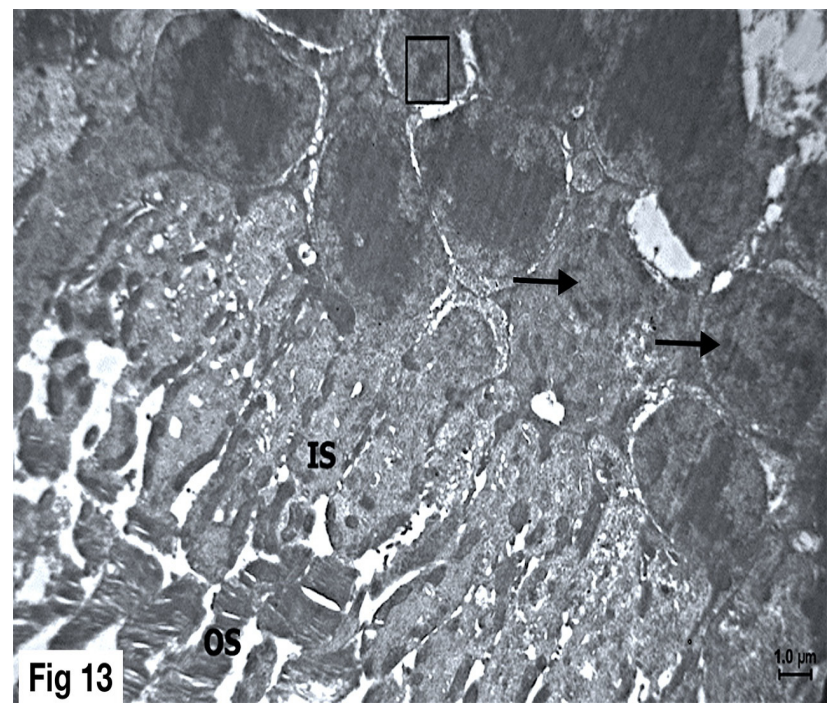

Figure 13: An electron micrograph of ultrathin section of photoreceptors of Group 3 showing improved histological features with regular outer segment [OS] and inner segment [IS]. Few nuclei of outer nuclear layer are shrunken [square] and others show dispersed chromatin [arrows].

[X 4000]

\section{DISCUSSION}

Methanol toxicity is extremely hazardous, it can cause toxic injury to the retina and optic nerve that may result in blindness ${ }^{[17,18]}$. Though the effect of methanol on the eye was extensively studied clinically, only few authors were interested to study methanol histological and ultrastructural effects on the retina. Our study offers an extensive detailed light and electron microscopic study, helping better understanding the effect of methanol on retina and giving histological basis for the clinical findings noticed by many authors.

In the present study, histological examination of the retina of rats treated with methanol showed marked edema of all layers, disorganized outer and inner nuclear layers as well as decreased number of ganglion cells. Electron microscopic examination revealed fragmented outer segment, disorganized inner segment. The most prominent ultrastructural observation in the retina was the decrease number and abnormal morphology of mitochondria in inner segment of photoreceptors.

Clinical, funduscopic and electroretinogram outcomes of methanol toxicity on the eye, could be better explained on histopathologic basis we observed. Optic nerve atrophy and cupping that were mentioned by many authors and suggested to be due to loss of ganglion cell[19, 20], this suggestion was justified in our study where ganglion cell damage appeared evident.

Regarding the mechanism of methanol toxicity, photoreceptors are highly metabolically active ${ }^{[21,22]}$. Formic acid, the toxic metabolite of methanol has been postulated to induce retinal and optic nerve toxicity by disrupting mitochondrial energy production as a result of its inhibitory influence on cytochrome oxidase enzyme of the electron transport chain. Some studies showing decreased retinal ATP concentrations in methanol intoxicated rats support the hypothesis that formic acid acts as a mitochondrial toxin. Other studies have revealed that methanol intoxication augment oxidative stress and decrease in the level of antioxidants. Both reduction of retinal ATP concentrations and decreased glutathione concentration have been considered to play a crucial role in the pathogenesis of methanol toxicity ${ }^{[23]}$. The proposed mechanism of methanol induced retinal toxicity is in accordance with the obvious mitochondrial damage observed in retinal ultrastructure observed in our current research.

Ethanol is widely used for management for methanol toxicity as it has been found to decrease the action of alcohol dehydrogenase on methanol by means of competitive inhibition; so methanol is eliminated by the kidneys rather than being converted in toxic metabolites. This is due to the fact that alcohol 
dehydrogenase affinity to ethanol is greater than methanol[24].

Similarly, to methanol toxicity, the efficacy of ethanol in treatment of methanol toxicity was only discussed clinically with only few microscopic data demonstrating ethanol abilities to prevent histopathological changes induced by methanol.

In the current work, administration of ethanol in methanol intoxicated rats resulted in preservation of normal retinal structure, with marked attenuation of methanol induced retinal damage at both histological and ultrastructural level providing a solid histological proof for ethanol clinical efficacy demonstrated in several studies.

Though some authors ask for considering the safety profile and therapeutic drug monitoring when choosing ethanol as antidote for methanol ${ }^{[25]}$, many authors found ethanol administration effective and safe in management of patients with methanol poisoning ${ }^{[24]}$.

Again, ethanol shows advantages over other inhibitors of alcohol dehydrogenase as fomepizole, which use is limited due to its high cost and lack of availability especially in developing countries where methanol poisoning is more likely to occur ${ }^{[26]}$.

\section{CONCLUSION}

Methanol intoxication can induce noticeable damage of the retina as shown by histological and ultrastructural examination. The administration of ethanol protects the retina and markedly reduces histopathological changes induced by methanol.

\section{CONFLICT OF INTEREST}

The authors have no conflicting financial interest.

\section{REFERENCES}

1. Nand L, Chander S, Kashyap R, Gupta D, Jhobta A. Methyl alcohol poisoning: a manifestation of typical toxicity and outcome. J Assoc Physicians India 2014;62(8):756-9.

2. Hassanian-Moghaddam H, Nikfarjam A, Mirafzal A, Saberinia A, Nasehi AA, Masoumi Asl H, et al. Methanol mass poisoning in Iran: role of case finding in outbreak management.: J Public Health (Oxf) 2015;37(2):354-9.

3. Barceloux DG, Bond GR, Krenzelok EP,Cooper H, Vale JA. American Academy of Clinical Toxicology practice guidelines on the treatment of methanol poisoning. J Toxicol Clin Toxicol 2002;40(4):415-46.
4. Hantson P, Haufroid V, Mahieu P. Survival with extremely high blood methanol concentration. Eur J Emerg Med. 2000;7(3):237-40.

5. Treichel JL, Henry MM, Skumatz CM, Eells JT, Burke JM. Formate, the toxic metabolite of methanol, in cultured ocular cells. Neurotoxicology. 2003;24(6):825-34.

6. Eells JT, Henry MM, Summerfelt P, Wong-Riley MT, Buchmann EV, Kane M, Whelan NT, Whelan HT. Therapeutic photobiomodulation for methanolinduced retinal toxicity. Proc Natl Acad Sci U S A. 2003 18;100(6):3439-44.

7. Hantson PE. Acute methanol intoxication: physiopathology, prognosis and treatment. Bull Mem Acad R Med Belg. 2006;161(6):425-34.

8. Paula EM, Mathangi DC, Namasivayam A. Free radical changes in methanol toxicity. Indian $\mathrm{J}$ Physiol Pharmacol 2003; 47:207-11.

9. Gonza'lez-Quevedo A, Obrego'n F, Urbina M. Effect of chronic methanol administration on amino acids and monoamines in retina, optic nerve, and brain of the rat. Toxicol Appl Pharmacol 2002; 185:77-84.

10. Fujihara M, Kikuchi M, Kurimoto Y. Methanol-induced retinal toxicity patient examined by optical coherence tomography. Jpn J Ophthalmol. 2006;50(3):239-41.

11. Sharma R, Marasini S, Sharma AK, Shrestha JK, Nepal BP. Methanol poisoning: ocular and neurological manifestations. Optom Vis Sci 2012; 89(2):178-82.

12. Garner CD, Lee EW, Terzo TS, Louis-Ferdinand RT. Role of retinal metabolism in methanol-induced retinal toxicity. J Toxicol Environ Health 1995;44(1):43-56.

13. Dudka J. The total antioxidant status in the brain after ethanol or 4-methylpyrazole administration to rats intoxicated with methanol. Exp Toxicol Pathol 2006; $57: 445-8$

14. Metz B., Kersten G. F. A., Hoogerhout P. Identification of formaldehyde-induced modifications in proteins: reactions with model peptides. J. Biol. Chem. 2004;279(8):6235-6243.

15. Bancroft JD, Gamble MN. Theory and Practice of Histological Techniques. 5th ed., ChurchillLivingstone, London, Edinburgh 2002;175.

16. Hanker J, Giammara B. Microwave-accelerated cytochemical stains for the image analysis and the electron microscopic examination of light microscopy diagnostic slides. Scanning 1993;15:67-80. 
17. Seme MT, Summerfelt P, Henry MM, Neitz J, Eells JT. Formate induced inhibition of photoreceptor functions in methanol intoxication. J Pharmacol. Exp. Ther 1999;289:361-70.

18. Eells JT, Henry MM, Summerfelt P, Wong-Riley MT, Buchmann EV, Kane M, et al. Therapeutic photobiomodulation for methanol-induced retinal toxicity. Proc. Natl Acad Sci USA 2003;100:3439-44.

19. Shin YW, Uhm KB. A case of optic nerve atrophy with severe disc cupping after methanol poisoning. Kor J Ophthalmol 2011; 25(2):146-50.

20. Moschos MM, Gouliopoulos NS, Rouvas A, Ladas I. Vision loss after accidental methanol intoxication: a case report. BMC Res Notes 2013;6:479.

21. Yang F, Yang CH, Wang FM, Cheng YT, Teng CC, Lee LJ, Yang CH, Fan LS. A high-density microelectrodetissue-microelectrode sandwich platform for application of retinal circuit study. Biomed Eng Online. 2015 26;14:109.
22. Reidel B, Thompson JW, Farsiu S, Moseley MA, Skiba NP, Arshavsky VY. Proteomic profiling of a layered tissue reveals unique glycolytic specializations of photoreceptor cells. Mol Cell Proteomics. 2011;10(3):M110.002469.

23. Seme MT, Summerfelt P, Neitz J, Eells JT, Henry MM. Differential recovery of retinal function after mitochondrial inhibition by methanol intoxication. Invest Ophthalmol Vis Sci 2001;42:834-41.

24. Danica L, Michael T, Garth D. Effectiveness of IV Ethanol Therapy Combined with Hemodialysis in the Treatment of Methanol and Ethylene Glycol Poisoning. Can J Hosp Pharm 2005;58:142-7.

25. Wedge MK, Natarajan S, Johanson C, Patel R, Kanji $\mathrm{S}$. The safety of ethanol infusions for the treatment of methanol or ethylene glycol intoxication: an observational study. CJEM 2012;14(5):283-9.

26. McMartin K, Jacobsen D, Hovda KE. Antidotes for poisoning by alcohols that form toxic metabolites. $\mathrm{Br}$ J Clin Pharmacol 2016;81(3):505-15. 